\title{
Orphan Diseases - die Waisenkinder erwachen
}

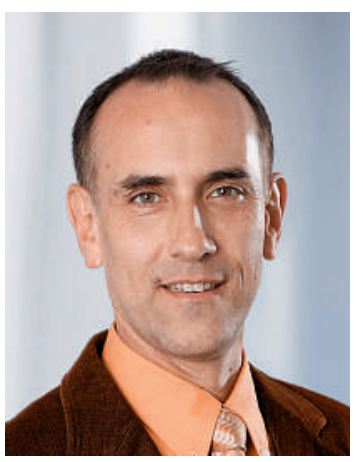

Unter Orphan Diseases werden Krankheiten subsummiert, die so selten auftreten, dass sie in einer Grundversorger-Praxis üblicherweise höchstens einmal pro Jahr vorkommen. In Zahlen bedeutet das für Europa eine relative Prävalenz von weniger als 5 Patienten pro 10000 Einwohner. Etwa 6000 bis 8000 dieser Erkrankungen sind heute bekannt; lange Zeit standen sie jedoch im Abseits der Medizin und hatten somit einen «Waisen»-Status.

Orphan Diseases bilden eine sehr heterogene Gruppe von zumeist komplexen Krankheitsbildern: Sie verlaufen chronisch, gehen mit Invalidität und eingeschränkter Lebenserwartung einher und führen häufig bereits im Kindesalter zu Symptomen. Etwa 80 Prozent der seltenen Erkrankungen sind genetisch bedingt oder mitbedingt, selten sind sie heilbar.

\section{Auch bei den Orphan Diseases besteht Handlungsbedarf.}

Patienten mit seltenen Erkrankungen und ihre Angehörigen sind mit einer Vielzahl von Schwierigkeiten und Problemen konfrontiert: Oft haben sie eine jahrelange Odyssee durch das Versorgungssystem hinter sich oder fühlen sich mit der Diagnose alleingelassen; gesicherte Diagnoseverfahren und Kenntnisse über die Erkrankung fehlen; vielfach fehlt eine adäquate medikamentöse Behandlung. Die Komplexität und Heterogenität der Orphan Diseases werden in den derzeitigen Vergütungssystemen meist nicht adäquat abgebildet.

Es ist evident, dass grosser Handlungsbedarf besteht. Nachhaltige Verbesserungen in Prävention, Diagnostik und Therapie können jedoch nur dann erreicht werden, wenn es gelingt, Initiativen zu bündeln und ein gemeinsames, koordiniertes und zielorientiertes Handeln aller Akteure des Gesundheitswesens, der Industrie und der Politik sowie der Patientenorganisationen zu bewirken.

Länder wie Frankreich, Italien, Spanien, Rumänien und Portugal haben bereits eine Strategie für seltene Erkrankungen erarbeitet. Um diese Entwicklung voranzutreiben, hat die EU-Kommission EUROPLAN initiiert. Was sich zu diesem Thema bisher in der Schweiz ereignete? Im August 2011 wurde die Interessengemeinschaft IG Seltene Krankheiten unter Beteiligung der FMH gegründet. Zum einen verfolgt die
IG zentrale strategische Ziele wie z. B. die Unterstützung und politische Begleitung der angestrebten nationalen Strategie im Bereich der seltenen Krankheiten, ein nationales Registrierungsprogramm zur Wissenssicherung und -vermittlung über Diagnose, Verlauf und Behandlung von seltenen Krankheiten und die Schaffung nationaler Kompetenzzentren in Zusammenarbeit mit europäischen / internationalen Referenzzentren und -netzwerken. Zum anderen strebt die IG auch einen «Runden Tisch» mit allen massgeblichen Akteuren als Austausch- und Koordinationsplattform an, will die Politik, Verwaltung, Medien und Öffentlichkeit für die Thematik «Seltene Krankheiten und Zugang zu Diagnose und Therapien» sensibilisieren und die nationale Strategie sowie den Massnahmenplan begleiten.

\section{Die Politik, Medizin und Wissenschaft sensibilisieren für Orphan Diseases und die Bedürfnisse von Patienten, das ist ein Ziel der IG Seltene Krankheiten.}

Mittlerweile ist das Thema auch auf der Agenda der Politik, zwei Round Tables mit hochkarätiger Besetzung fanden letztes Jahr statt. Es bewegt sich etwas - die Waisenkinder der Medizin rücken endlich weg von ihrem Schattendasein. Es gibt jedoch noch viel zu tun:

- Wegen der Heterogenität der Orphan Diseases und dem Wissensstand dürften Bottom-up-Ansätze der Forschungsförderung am vielversprechendsten sein.

- Die Überführung von Erkenntnissen und Ergebnissen der Grundlagenforschung in die klinische Anwendung und in klinisch relevantes Wissen zum Patientennutzen ist zu verbessern (Translation «bench to bedside $\&$ back»).

- Die translationale Forschung muss interdisziplinär arbeiten und benötigt Vernetzung von (Grundlagen-)Wissenschaftlern und Klinikern - national wie international.

- Aufgrund limitierter Ressourcen ist eine sinnvolle Vernetzung angezeigt wie z. B. das gemeinsame Nutzen von Infrastrukturen, Daten- und Materialbanken, Technologieplattformen. Netzwerke müssen somit gefördert werden, sowohl auf nationaler als auch auf internationaler Ebene! Entscheidend ist, was am Ende beim Patienten ankommt. Und da besteht noch erheblicher Verbesserungsbedarf!

Dr. med. Gert Printzen, Mitglied des Zentralvorstandes, Verantwortlicher Ressort Heilmittel 